\title{
AmAZONANAS
}

Revista científica del Amazonas

Volumen 3 Número 5

Enero-junio 2020

\section{Doença diarreica aguda em menores de 5 anos em um hospital da fronteira do Brasil}

\section{Acute diarrheal disease in children under 5 years old in a Brazilian border hospital}

Recibido: 13 de julio 2019
Aceptado: 9 de septiembre de 2019

Escrito por:

Heluza Monteiro De Oliveira ${ }^{5}$

Kênia Richlli Barros Gomes ${ }^{6}$

Cecília Rafaela Salles Ferreira ${ }^{7}$

Caroline Raissa Salles Ferreira ${ }^{8}$

https://orcid.org/0000-0003-2591-0011

Veridiana Barreto do Nascimento ${ }^{9}$

http://orcid.org/0000-0003-4655-9670

Wanderson Willian Dos Santos Dias ${ }^{10}$

\section{Resumo}

Objetivo: analisar a frequência de doenças diarreicas agudas (DDA) em crianças de 0 a 5 anos de idade assistênciado no período de janeiro a dezembro de 2017 no Hospital Estadual situado na fronteira franco brasileira. Materiais e Métodos: pesquisa documental com abordagem quantitativa. A coleta de dados ocorreu a partir dos dados obtidos no Serviço de Arquivo Médico e Estatística do Hospital Estadual de Oiapoque, em prontuário, levando em consideração as seguintes variáveis: Sexo, idade, história pregressa, peso, sinais e sintomas, identificação do tipo de diarreia (com sangue ou não), exames laboratoriais e manejo farmacológico e não farmacológico. Resultados: A amostra foi constituída por 602 prontuários analisados de paciente atendidos na faixa etária de 0 mês à 5 anos com sinais e sintomas de DDA. Dos 602 pacientes, 60 (10\%) foram internados no período do estudo, com confirmação diagnóstica de DDA com sintomas de desidratação e $8(1,3 \%)$ pacientes com diarreia associado a pneumonia, com idades de 1 mês e 14 dias a 5 anos de idade. Conclusão: Os dados obtidos estão de encontro com a literatura que aponta para fatores atrelados a ocorrência da doença, aspectos socioeconômicos, culturais, e neste caso principalmente ambientais e de infraestrutura, com problemas comuns que ainda afetam os países subdesenvolvidos ou em desenvolvimento.

Palavras-chave: Diarreia, Diarreia infantil, Atenção Integrada às Doenças Prevalentes na Infância.

\footnotetext{
${ }^{5}$ Universidade Federal do Amapá, Docente da Universidade Federal do Amapá - Campus Binacional do Oiapoque, Macapá, Amapá, Brasil

${ }^{6}$ Universidade Federal do Amapá, Acadêmica de enfermagem, Macapá, Amapá, Brasil

${ }^{7}$ Universidade Federal do Amapá, Divisão Básica de Saúde, Macapá, Amapá, Brasil

${ }^{8}$ Universidade Federal do Amapá, Mestrado em Ciências da Saúde, Macapá, Amapá. Brasil

${ }^{9}$ Universidade Federal do Amapá, Enfermagem, Docente da Universidade Federal do Amapá - Campus Binacional do Oiapoque, Amapá, Brasil

${ }^{10}$ Universidade Federal do Amapá, Enfermagem, Médico Residente Do Grupo Hospitalar Conceição - Porto Alegre, RS, Brasil
} 


\title{
AMAZUNANAS
}

\begin{abstract}
Objective: To analyze the frequency of acute diarrheal diseases (ADD) in children from 0 to 5 years of age assisted from January to December 2017 at the State Hospital located on the Brazilian border. Materials and Methods: documentary research with quantitative approach. Data collection occurred from the data obtained from the Medical and Statistical Archive Service of Oiapoque State Hospital, in medical records, taking into consideration the following variables: Sex, age, previous history, weight, signs and symptoms, identification of the type of diarrhea (blood or not), laboratory tests and pharmacological and non-pharmacological management. Results: The sample consisted of 602 patient records analyzed from 0 months to 5 years old with signs and symptoms of DDA. Of the 602 patients, 60 (10\%) were admitted during the study period, with diagnostic confirmation of DDA with symptoms of dehydration and $8(1.3 \%)$ patients with pneumonia-associated diarrhea, aged 1 month and 14 days to 5. years old. Conclusion: The data obtained are in agreement with the literature that points to factors linked to the occurrence of the disease, socioeconomic, cultural aspects, and in this case mainly environmental and infrastructure, with common problems that still affect underdeveloped or developing countries.
\end{abstract}

Keywords: Diarrhea, Infantile diarrhea, Integrated Attention to Prevalent Diseases in Childhood.

\section{Introdução}

As doenças diarreicas agudas (DDA) são síndromes clínica multifatorial causada por bactérias, vírus, fungos e/ou parasitas, que têm como características predominantes o aumento no número de evacuações (frequência), com fezes de consistência diminuída, podendo ser liquida ou aquosa, com inicio repentino e duração de até duas semanas (Façanha; Pinheiro, 2005; Brasil, 2017).

As DDA têm como principais complicações a desidratação, a gravidade depende da presença e intensidade da desidratação ou do tipo de toxina produzida pelo patógeno que pode provocar outras complicações. Com frequência vem acompanhada de outros sintomas como febre acima de $38{ }^{\circ} \mathrm{C}$, vômitos, cólicas e algia abdominal, podendo apresentar muco e sangue (UNCF, 2016).

Do ponto de vista de importância epidemiológica, em 2015 mais de 300 mil crianças menores de 5 anos morreram por infecção em decorrência de DDA no mundo. Essas mortes podem ser atribuídas a práticas de higiene corporal e alimentar precárias, abastecimento inseguro de água para beber e acesso inadequado a instalações de saneamento (Porcy et al., 2013).

As DDA em território brasileiro ocupam o terceiro lugar como causa de mortalidade em crianças menores de cinco anos e o segundo lugar em menores de até 01 (um) ano de idade, sendo que a maioria das crianças, que se encontram-se em baixas condições socioeconômicas. Os estados da Amazônia Legal ainda mantêm indicadores preocupantes de ocorrências de DDA, onde $30 \%$ das internações de crianças são em decorrência de DDA (Viana; Freitas; Griatti, 2016). 


\section{AMAZÚNAS}

A prevalência de DDA não é um problema novo de saúde no Brasil. Na região Norte o risco de morte por DDA é de quatro a cinco vezes maior do que na região Sul, representando cerca de $30 \%$ do total de mortes durante o primeiro ano de vida. No Norte do país a maioria das cidades não tem serviços de saneamento básico e população apresentar níveis socioeconômicos baixos, sendo estes fatores determinantes para as DDA (Farias; Paz, 2016; Asmus; Seixas; Gonzalez, 2017).

Esses elevados números de casos de DDA e os casos de mortalidade da região Norte evidenciam a problemática do estudo, pois o município de abrangência do estudo está localizado dentro da Amazônia legal, especificamente o município do Oiapoque no estado do Amapá, com características que tornam a população vulnerável as DDA, tais como: inexistência de saneamento básico, lixão a céu aberto, sistema de abastecimento de agua ineficaz e oferta baixa de serviços de saúde na atenção básica.

O município de Oiapoque está situado no extremo norte do estado do Amapá, possui área de fronteira, limita-se ao norte com a Guiana Francesa, além dos municípios de seu entorno e quatro distritos, tem ainda, distribuídos sua em área geográfica municipal, áreas indígenas localizadas na Br 156, ao longo dos rios: Uaçá, Curipi e Oiapoque, a população indígena compreende cerca de $30 \%$ da população total do município, é previsto pela Política Nacional de Atenção aos Povos Indígenas a redução de agravos a saúde infantil, entre elas as doenças diarreicas. Segundo Boletim epidemiológico, publicado pelo Ministério da Saúde, em Oiapoque/ Amapá, entre as semanas epidemiológicas 09 e 17 do ano de 2015 ( 28 de fevereiro a 30 de abril) foram notificados 219 pacientes com DDA, já em 2016 houve um aumento significante para 634 casos. Assim, este estudo tem como hipótese que há uma elevada prevalência de número de casos DDA no referido município (Brasil, 2018; Brasil, 2016).

O presente artigo tem-se como objetivo geral analisar a frequência de doenças diarreicas agudas (DDA) em crianças de 0 a 5 anos de idade assistênciado no período de janeiro a dezembro de 2017 no Hospital Estadual situado na fronteira franco brasileira.

\section{Material e método}

Os dados apresentados são oriundos de uma pesquisa documental, de caráter exploratório com abordagem quantitativa. A coleta de dados ocorreu a partir dos dados obtidos no Serviço de Arquivo Médico e Estatística (SAME) do Hospital Estadual de Oiapoque (HEO).

As variáveis utilizadas para o estudo foram oriundas do prontuário medico da criança, sendo levar em consideração as seguintes variáveis: Sexo (se masculino ou feminino), idade (em faixa etária), história pregressa, peso $(\mathrm{kg})$, sinais e sintomas, identificação do tipo de diarreia (com sangue ou não), exames laboratoriais e manejo farmacológico e não farmacológico.

A população foi do tipo censo, contemplando todos os dados do período do estudo. Quanto aos critérios de inclusão: Foram incluídos na pesquisa todos os boletins de atendimentos, registros e prontuários pediátricos de menores de 0 a 5 anos de idade acometidas por doença diarreica aguda, no período de janeiro a dezembro de 2017, sendo 


\section{AMAZUNANAS}

a coleta de dados realizada em 2018. Foram captados 639 prontuários, seguindo o critério de exclusão: dados incompletos e/ou falta de informações, a amostra consistiu em 602 casos analisados de paciente atendidos na faixa etária de 0 mês a 5 anos com sinais e sintomas de DDA.

Para análise da coleta, foi criada uma planilha no Excel-2010 para tabulação dos dados, sendo posteriormente transferida para o Statistical Package for Social Sciences (SPSS), versão 22.0 for Windows (IBM. SPPS STATISTICS 22). Para caracterização das variáveis do estudo, utilizou-se somente frequências absolutas e relativas, para as variáveis idade e tempo de internação foram realizadas a média e desvio padrão.

Pesquisa foi norteada de acordo com os padrões éticos da Resolução que rege a integralmente as diretrizes e referencias dispostos na resolução 466/2012 do Conselho Nacional de Saúde, que trata da regulamentação de pesquisas envolvendo seres humanos. Por se tratar de um banco de dados restrito foi considerado aceito e pronto para ser executada após a emissão da Declaração de Aceite Institucional por membro, diretora do hospital e equivalente responsável coordenador do setor SAME do hospital. A pesquisa foi aprovada com número do parecer: 2.626.146 do Comitê de Ética da Universidade Federal do Amapá vinculado a Plataforma Brasil.

\section{Resultados}

A amostra foi constituída por 602 prontuários analisados de paciente atendidos na faixa etária de 0 mês 5 anos com sinais e sintomas de DDA. Dos 602 pacientes, 60 (10\%) foram internados no período do estudo, com confirmação diagnóstica de DDA com sintomas de desidratação e $8(1,3 \%)$ pacientes com diarreia associado a pneumonia, com idades de 1 mês e 14 dias a 5 anos de idade.

Na Tabela 1, observa-se o perfil das crianças acometidas por DDA. Verifica-se que a maioria era do sexo masculino com 311(52\%), em relação à faixa etária prevalente foi de 1 ano a 1 ano e 11 meses com $169(28 \%)$.

\section{Tabela 1.}

Perfil das crianças acometidas Diarreia Aguda. Oiapoque/AP. 2019.

\begin{tabular}{llll}
\hline Variavéis & & fn & $\begin{array}{l}\mathrm{f} \\
\%\end{array}$ \\
\hline Sexo & Feminino & 291 & 48 \\
& Masculino & 311 & 52 \\
Faixa etária & 0 a 11 meses & 129 & 22 \\
Média=2,93;DP=0,70) & 1 ano a 1 ano e 11 meses & 169 & 28 \\
& 2 anos a 2 anos e 11 meses & 122 & 20 \\
& 3 anos a 3 anos e 11 meses & 68 & 11 \\
& 4 anos a 4 anos e 11 meses & 65 & 11 \\
& 5 anos & 49 & 8 \\
\hline
\end{tabular}

Fonte: Formulário de coleta de dados, 2019. 
Em relação aos meses de maior prevalência de casos de DDA, os maiores registros ocorreram nos meses de janeiro com 119 (20\%), maio com 58 (10\%), e dezembro com $67(11 \%)$ (Tabela 2).

Tabela 2.

Caracterização do percentual de casos por mês de janeiro a dezembro de 2017. Oiapoque, AP, 2019.

\begin{tabular}{lll}
\hline Meses & fn & f\% \\
\hline Janeiro & 119 & 20 \\
Fevereiro & 48 & 8 \\
Março & 43 & 7 \\
Abril & 43 & 7 \\
Maio & 58 & 10 \\
Junho & 38 & 6 \\
Julho & 31 & 5 \\
Agosto & 28 & 5 \\
Setembro & 50 & 8 \\
Outubro & 32 & 5 \\
Novembro & 45 & 8 \\
Dezembro & 67 & 11 \\
\hline
\end{tabular}

Fonte: Instrumento de Coleta, 2019.

Na tabela 3 com relação ao período de internação hospitalar, 21 (31\%) pacientes, que representa tiveram de 1 a 2 dias de internação, 41 (60\%) tiveram de 3 a 4 dias e apenas 9 $(6 \%)$ de 5 a 6 dias de internação.

No que se refere ao sinal de desidratação dos pacientes atendidos, observa-se que 170 (30\%) das crianças apresentaram desidratação. No tocante, as características de peso das crianças 559 (93\%) estavam apresentando peso adequado para a faixa etária de idade, enquanto 16 (3\%) apresentavam baixo peso e $27(4 \%)$ estavam acima do peso para idade. Em relação a óbitos dos pacientes que apresentaram quadro diarreico, não houve ocorrência associada a este, apenas um caso devido patologia crônica associada.

Tabela 3.

Caracterização das variáveis clinicas. Oiapoque, AP, 2019.

\begin{tabular}{|c|c|c|c|}
\hline es & & Fn & $\mathrm{f} \%$ \\
\hline \multirow{2}{*}{ Óbito } & Sim & 1 & - \\
\hline & Não & 601 & 100 \\
\hline \multirow[t]{2}{*}{ Realizou exames } & Sim & 75 & 12 \\
\hline & Não & 527 & 88 \\
\hline \multirow[t]{2}{*}{ Terapia de Reidratação oral } & Sim & 171 & 28 \\
\hline & Não & 431 & 72 \\
\hline Tempo de internação & 1 a 2 dias & 21 & 31 \\
\hline \multirow[t]{2}{*}{ 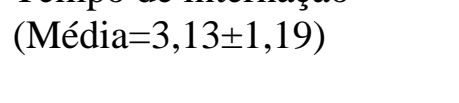 } & 3 a 4 dias & 41 & 60 \\
\hline & 5 a 6 dias & 6 & 9 \\
\hline
\end{tabular}




\section{AMAZÖNAS}

\begin{tabular}{llll}
\hline Hidratação Corporal & Não informado & 420 & 70 \\
& Desidratação & 170 & 30 \\
& Desidratação/apatica & 1 & - \\
& Desidratação/Hipo & 1 & - \\
& Anorexia & 1 & - \\
Peso por idade & Adequado & 559 & 93 \\
& Baixo Peso & 16 & 3 \\
& Peso Elevado & 27 & 4 \\
\hline
\end{tabular}

Fonte: Instrumento de Coleta, 2019.

Legenda: (-) = sinal convencional utilizado; dado numérico igual a zero não resultante de arredondamento.

No que tange a manifestação clinica de sangue nas fezes, apenas $4 \%$ apresentaram essa manifestação (tabela 4).

Tabela 4.

Sinais e sintomas: Fezes com sangue. Oiapoque, AP. 2019.

\begin{tabular}{llll}
\hline Variáveis & & fn & f\% \\
\hline Diarréia com sangue & Sim & 23 & 4 \\
& Não & 579 & 96 \\
\hline
\end{tabular}

Fonte: Instrumento de Coleta, 2018.

\section{Discussao}

O número de casos de DDA me crianças menores de 5 anos demonstra a importância da discussão da temática para os países subdesenvolvidos, uma vez que apresentam fatores de vulnerabilidade para o desenvolvimento de processo patológico no público infantil, com isso ressalta-se a importância de buscar medidas preventivas e terapêuticas que sejam implementadas, com decisões de ações direcionadas para essas populações, buscando reduzir o número de casos.

Os dados relatados apontam maiores casos nos primeiros anos de vida, isto é preocupante, principalmente pelos quadros de desidratação e pneumonia associados. $\mathrm{O}$ quantitativo de casos identificados $(n=602)$ levanta sobretudo questões de relevância para o âmbito da saúde pública, quadros de DDA estão intimamente ligados ao saneamento básico. A adoção de medidas de saneamento básico apresentou no mundo um impacto importante no declínio da morbidade por doenças da infância, entre elas a diarreia, que possui importante veículo de transmissão água contaminada e ausência de esgoto (França et al., 2017).

No Amapá, atualmente, o índice de mortalidade infantil se apresenta inferior a $25 \%$ de óbitos a cada mil nascidos vivos entre os estados que compõe a região norte, e por apresentar as maiores taxas de morbimortalidade infantil por doenças evitáveis. Se comparado ao estado do Acre que apresenta declínio no número de casos, resultados 


\section{AMAZÓNAS}

atrelados a melhora em saneamento básico neste estado, o Amapá apresenta um comportamento linear, sem melhoras no quadro epidemiológico (Cesário et al., 2006).

Outra característica do perfil é quanto o maior número de casos em crianças do sexo masculino. $\mathrm{O}$ gênero masculino também foi prevalente em outro estudo desenvolvido no Acre na investigação de prevalência de DDA, não sendo encontrado na literatura associação entre o sexo e a ocorrência da DDA, no entanto, essa caracterização demonstra a necessidade de educação em saúde sobre higiene a este público (Gomes et al., 2017).

Uma variável de relevância no estudo é a ocorrência de DDA em crianças menores de 3 anos. Nessa faixa etária as crianças podem se contaminar através de objetos, contato pessoa-a-pessoa, geralmente em creches e/ou escolas; e ainda, apresentam menor resposta imunológica diante de determinantes como viroses e o uso indiscriminado de água não tratada presente nas minas existentes na região em que moram (Miyashi et al., 2013).

Do total das 602 crianças notificadas com DDA, 420 tem menos de 3 anos de vida. É necessário mencionar a importância dos cuidados com a alimentação das crianças nos primeiros anos de vida. De acordo, com a Organização Mundial da Saúde, os bebês, nos seis primeiros meses de vida, devem se alimentar exclusivamente de leite materno. Para a instituição, apenas após meio ano de vida é que devem ser introduzidos outros alimentos, como frutas e papinhas, porém, o aleitamento materno ainda deve ser predominante (OMS, 2017).

Em relação aos meses com maiores casos de DDA ocorreram nos meses de dezembro a janeiro. Este resultado pode estar relacionado aos fatores ambientais, na região norte, a pluviosidade na maioria dos meses do ano é significativa, as temporadas de chuvas iniciam no mês de dezembro e se estende até maio, com a chegada das chuvas, os casos de DDA aumentam, trazendo com ela a proliferação de vírus e bactérias prejudicando a saúde humana.

No período chuvoso (dezembro a maio) foram registrados 378 casos, o que corresponde a $63 \%$ dos casos de diarreia aguda. Este dado corrobora com o trabalho realizado no estado do Pará, que tem os maiores índices de mortalidade devido a quadro diarreico ocorreram no período chuvoso (janeiro a maio). A associação do aumento do índice de contaminação bacteriológica de águas de poços do lençol freático superficial está associada com as chuvas, assim essa piora da qualidade da água pode esta associada ao escoamento das águas da chuva carreando excretas humanas e animais, o uso dessa água não tratada aumentaria a frequência de diarreias no período chuvoso (Dias et al., 2010; Gomes; Mota; Damacena, 2014).

Desta forma, reafirma-se que a DDA constitui um problema de saúde pública que está associado a fatores ambientais. Como é de interesse da saúde preventiva, a raiz do problema é que deve ser analisada, ou seja, os aumentos das DDA estão relacionados desde questões sociais, econômicas, até políticas, além de questões climáticas como a pluviosidade, sendo este um dos fatores que possa está relacionado ao número de casos no presente estudo. 
Diante disso, empregar medidas de saneamento básico para a população de uma região, resulta em diminuição do número de casos de DDA. O saneamento é uma das principais intervenções que podem ser utilizadas para combate este problema de saúde pública (Farias; Paz, 2016).

Para o diagnostico etiológico, é importante a realização de exames, neste estudo, $88 \%$ não realizaram exame para auxiliar na investigação do agente etiológico. A ausência de realização do exame demonstrou ser um entrave para pesquisa, principalmente por não descreve informações que esclareça o motivo do não registro, demonstrando a necessidade de abrangência de outros estudos na área (SBP, 2017; Brandt; Antunes; Silva, 2015).

Dos patógenos que são associados a este agravo estão os parasitas, e bactérias, além do vírus, em especial como rotavírus, norovírus e astrovírus. Os agentes etiológicos para a DDA tem sua principal forma de transmissão a via oral-fecal, através da contaminação de alimentos agua e objetos (Silva et al., 2013; Sousa et al., 2016).

Uma investigação da prevalência de enteropatogenos bacterianos na DDA, aponta como os agentes etiológicos mais frequentes a Escherichia coli diarreiogênicas, Campylobacter jejuni e Campylobacter coli. Shigella spp e Salmonella spp, estes agentes então presentes em água de rios, enseadas, lagoas e região portuária (Silva et al., 2013; Sousa et al., 2016). Tendo em vista que na região da pesquisa a população vivencia suas práticas cotidianas em entorno de um rio, infere-se que este pode ser um dos agentes etiológicos presentes, além de que essa característica ambiental somada a precariedade do saneamento, podem ser fatores de vulnerabilidade para ocorrência DDA.

A Terapia de Reidratação Oral que consiste na reposição de fluidas como sódio e potássio, e constantemente associado ao uso do Zinco, entre outros componentes, que junto a Terapia de reidratação venosa (TRV), preconizados em 1970, é considerado um marco no tratamento da DDA. Nessa pesquisa, $70 \%$ não realizaram tratamento com TRO, um estudo que avalia as evidências no tratamento de DDA, descreve que apesar das evidências cientificas, onde os antibióticos devem ser utilizados para casos específicos, pouco se tem avançado para o uso da TRO, que deve ser o tratamento de primeira escolha (Brandt; Antunes; Silva, 2015).

Os riscos de desidratação também devem ser levados em considerações, recomendada tanto para o tratamento quanto para a prevenção, pois dependendo do nível de perda e desequilíbrio de eletrólitos, e o paciente pode entrar em pré-choque ou choque. Em quadros diarreicos deve-se priorizar a terapia da reidratação oral, utiliza-se a parenteral em casos de urgência, ou quando há perda exacerbada de agua e eletrólitos e risco de óbito. A reposição de líquidos é preconizado pelo ministério da saúde em casos de diarreia, independentemente da faixa etária (Brandt et al., 2015).

No tocante ao peso as crianças com DDA apresentavam peso adequado para a faixa etária. A criança que apresenta quadro diarreico tende a perder peso devido perda excessiva de fezes e líquidos e pode ter diminuição de apetite. É fundamental fazer o acompanhamento do peso da criança que apresenta diarreia aguda. Considera-se que 


\section{AMAZÓNAS}

perda de peso de até $5 \%$ represente a desidratação leve; entre $5 \%$ e $10 \%$ a desidratação é moderada; e perda de mais de $10 \%$ traduz desidratação grave e desnutrição (SBP, 2017).

Os sintomas evidenciados foram: diarreia, vômito, gripe e/ou febre e tosse, casos de doenças secundárias foram encontradas como a pneumonia. Conforme a OMS a DDA pode ser classificada em: aquosa, com duração de ate 14 dias que pode causar a desidratação e desnutrição e a diarreia aguda com sangue (disenteria), com presença de sangue das fezes, que foi evidenciada em $4 \%$ da amostra $(n=23)$ e geralmente é associada por infecção sistêmica e complicações como a desidratação, tendo como agente etiológico principal bactérias entre elas a Shigella, são definidas como agudas quadros com até 14 dias mas em geral duram até 7, o tratamento adequado pela equipe de saúde, respeitando os protocolos existentes, deve ser ofertado, para metas como a erradicação de mortes por DDA até 2030, incentivada pela OMS seja alcançada (SBP, 2017).

Como limitações do estudo, a realização de pesquisa que envolve dados registrados durante a rotina do serviço, pode apresentar informações incompletas, subnotificações, além de não possibilitar a investigação de outras variáveis que podem subsidiar a discursão. Ainda assim, a pesquisa em dados preexistentes possibilita a multiplicidade do método em outras regiões, e permeia a discursão com outras realidades. O elevado quantitativo de 602 casos de DDA no município, em uma análise generalizada, é reflexo da deficiência saneamento básico ainda vivenciadas em países não desenvolvidos.

\section{Conclusão}

A prevalência de 602 casos de DDA em crianças de 0 a 5 anos de idade foi identificada no município de estudo em um período de um ano, sendo crianças de menos de três anos as mais acometidas por esse agravo. Os dados obtidos estão de encontro com a literatura que aponta para fatores atrelados a ocorrência da doença, aspectos socioeconômicos, culturais, e neste caso principalmente ambientais e de infraestrutura, com problemas comuns que ainda afetam os países subdesenvolvidos ou em desenvolvimento, como a precariedade de saneamento básico. Como dificuldades encontradas, a não realização da investigação laboratorial do agente etiológico, assim como a pouca adesão ao tratamento de primeira escolha a Terapia de Reidratação Oral, demonstrando alguns entraves do tratamento que refletem que saúde pública da região se assemelha a de outras regiões do país. Como contribuição para pratica, os dados permeiam medidas de fortalecimento das ações de promoção da saúde e tratamento das DDA, e desperta a necessidade de outras pesquisas que demonstre outras realidades epidemiológicas para o fortalecimento de políticas publicas que reduzam a mortalidade infantil no país.

\section{"CONFLITO DE INTERESSES"}

Não há conflito de interesse entre os autores.

\section{Referência}

Asmus, F.G., Seixas, C.R.S. \& Gonzalez, E. 2007. Diarreias Agudas em Caraguatatuba: Situação Epidemiológica e Sugestões para Monitoramento. Saúde Meio Ambiente. 6(1):71-84. 
Brasil. Ministério da Saúde. 2017. Secretaria de Vigilância em Saúde. Coordenação-geral de Desenvolvimento da Epidemiologia em Serviços. Brasília.

Brasil. Ministério da Saúde. 2017. Doença Diarreica Aguda. Brasília.

Brasil. Ministério da Saúde. 2016. Coordenadoria de Vigilância em Saúde - CVS/AP. Grupo Técnico das Doenças de Veiculação Hídrica, Alimentar e do Solo. Brasília.

Brandt, K. G., Antunes, M. M. C. \& Silva, G. A. P. Diarreia aguda: manejo baseado em evidências. J Pediatr.91(6 Suppl.1), p.36-S43.

Cesario, R. R. \& Tavares-Neto, J. 2006. Prevalência de diarréia na população do Distrito Docente-Assistencial do Tucumã, Rio Branco, Estado do Acre, Brasil, em 2003. Epidemiol Serv Saude. 15(3), p.19-28.

Dias, D. M., Silva, A. P., Helfer, A. M., Maciel, A. M. T. R., Loureiro, E. C. B., Souza, C. O. 2010. Morbimortalidade por gastroenterites no Estado do Pará. Rev Pan-Amaz Saude. 1(1):53-60.

Dulgheroff, A. C. B., Figueiredo, E. F., Gouvêa, V. S., Domingues, A. L. S. 2014. Changes in epidemiology of rotavirus in the Triângulo Mineiro region of Brazil: lack of two consecutive rotavirus seasons. Braz J Med Biol Res, 47(12), p.1091-5.

Façanha, M. C. \& Pinheiro, A. C. 2005. Comportamento das doenças diarréicas agudas em serviços de saúde de Fortaleza, Ceará, Brasil, entre 1996 e 2001. Cad. Saúde Pública. 21(1):49-54.

Farias, A.M. \& Paz, M. C. F. 2016. Importância do Saneamento Básico na Prevenção de Doenças Diarreicas: Uma Revisão Integrativa. CONBRACIS, Campina grande.

França, E. B., Lansky, S., Rego, M. A. S., Malta, D. C., França, J.S., Teixeira, R., Porto, P., Almeida, M. F., Souza, M. F. M., Szwarcwald, C. L., Mooney, M., Naghavi. M., Vasconcelos, A. M. N. 2017. Principais causas da mortalidade na infância no Brasil, em 1990 e 2015: estimativas do estudo de Carga Global de Doença. Rev. bras. epidemiol. 20(Supl. 1):46-60.

Gomes, L. O., Matos, H. J., Silva, M. C. M., Loureiro, E. C. B., Macarenas, J. D’ARC. P., Gabbay, Y. B., Rocha, D. C. C. 2017. Aspectos epidemiológicos das enteroinfecções bacterianas em menores de 5 anos de idade em Rio Branco, estado do Acre, Brasil. Rev Pan-Amaz Saude. 8(4).

Gomes, I. C., Mota, M. L. \& Damacena, M. C. S. 2014. Epidemiologia das Doenças Diarreicas Agudas no CARIRI - CE. Revista Interfaces: Saúde, Humanas e Tecnologia.;2(2).

Miyashita, N., Akaike, H., Teranishi, H., Kawai, Y., Ouchi, K., Kato, T, et al. 2013. Diagnostic value of symptoms and laboratory data for pertussis in adolescent and adult patients. BMC Infect Dis. 13(1).

Porcy, C., Ferro, T. A. F., Monteiro, S. G., Menezes, R. A. O., Barbosa, F. H. F., Monteiro Neto, V. 2013. Epidemiologia da Doença Diarreica Associada às Escherichia coli Diarreiogênicas em Crianças Residentes em uma Área Alagada de Macapá - Amapá, Brasil. Ciencia equatorial 3(1):57-68.

OMS. Organização Mundial de Saúde. 2017. Protecting, promoting and supporting breastfeeding in facilities providing maternity and newborn services Guideline.

UNCF. United Nations Children's Fund. 2016. Levels and Trends in Child Mortality.

Silva, M. F., Gómez, M. M., Rose, T. L., Volotão, E. M., Carvalho-Costa, F. A., Bello, G. et al. 2013. Lineages of group A rotaviruses circulating over 20 years in Brazil: proposal of six different sub-lineages for P [8]-3 clade. Infect Genet Evol. 16, p.2005. 


\section{AMAZÓNAS}

Sociedade Brasileira de Pediatria. 2017. Diarreia aguda: diagnóstico e tratamento. Departamento Científico de Gastroenterologia.

Sousa, C. O., Melo, T. R. B., Melo, C. S. B., Menezes, E. M., Carvalho, A. C., Monteiro, L. C. R. 2016. Escherichia coli enteropatogênica: uma categoria diarreiogênica versátil. Rev Pan-Amaz Saude. 07(2).

Viana, R. L., Freitas, C. M. \& Giatti, L. L. 2016. Saúde ambiental e desenvolvimento na Amazônia legal: indicadores socioeconômicos, ambientais e sanitários, desafios e perspectivas. Saude soc. 25(1): 233-246. 